\title{
On ground state of spinor Bose-Einstein condensates
}

\author{
Daomin Cao, I-Liang Chern, and Jun-Cheng Wei
}

\begin{abstract}
We prove the existence of the ground state for the spinor Bose-Einstein condensates in the one-dimensional case.
\end{abstract}

Mathematics Subject Classification (2010). Primary 35J65;

Secondary 35B40, 35B45.

Keywords. Ground States, Bose-Einstein Condensates, Elliptic Systems.

\section{Introduction}

In 1925, Einstein predicted that massive non-interacting bosons at low temperature could occupy the same lowest-energy single-particle state and form the so-called Bose-Einstein condensates (BEC). This was realized experimentally in 1995 by laser cooling technique for several alkali atomic dilute gases, such as ${ }^{87} \mathrm{Rb}[1],{ }^{23} \mathrm{Na}$ [7], and ${ }^{7} \mathrm{Li}$ [5]. For bosonic atoms, the total spin number $F$ corresponding to the lowest energy state has to be an integer with $2 F+1$ hyperfine states $\left(m_{F}=-F,-F+1, \ldots, F-1, F\right)$. They are called spin- $F$ BEC. For the above alkali atoms, $F=1$.

In early experiments, the atoms are confined by magnetic trap, the spin direction follows the magnetic field and thereby the spin degree of freedom are frozen. The atomic gas is then described by a scalar wave function. Through the mean-field approximation, this wave function satisfies the Gross-Pitaevskii equation (GPE) [6,11,21]:

$$
i \hbar \partial_{t} \psi=\frac{\delta H}{\delta \psi^{*}}=\left[-\frac{\hbar^{2}}{2 m} \nabla^{2}+V(x)+U_{0}|\psi|^{2}\right] \psi .
$$

Here, $\hbar$ is the Planck constant, $m$ the mass, $\psi^{*}$ the complex conjugate of $\psi$, $V$ the trapping potential

$$
H(\psi)=\int_{\mathbb{R}^{d}} d x\left[\frac{\hbar^{2}}{2 m}|\nabla \psi|^{2}+V(x)|\psi|^{2}+\frac{U_{0}}{2}|\psi|^{4}\right]
$$


the Hamiltonian, and $d=1,2,3$ the underlying space dimensions. The parameter $U_{0}=4 \pi^{2} a_{0} / m$ is the effective pairwise interaction energy between atoms, and $a_{0}$ is the s-wave scattering length.

Recently, optical dipole trap is used to confine alkali atoms. Unlike the magnetic trap, all hyperfine states are active [3,10,18,22,23]. The theory for these spinor BEC was developed independently by several researchers $[12,13,20]$. In the case of $F=1$, the spin- 1 BEC are described by a vector wave function $\boldsymbol{\Psi}=\left(\psi_{1}, \psi_{0}, \psi_{-1}\right)^{T}$, where each component corresponds to the $m_{F}=1,0,-1$ hyperfine states, respectively. The governing equation is a generalized Gross-Pitaevskii equation (GGPE):

$$
i \hbar \partial_{t} \boldsymbol{\Psi}=\frac{\delta H}{\delta \boldsymbol{\Psi}^{\dagger}},
$$

where the Hamiltonian is given by

$$
H(\boldsymbol{\Psi})=\int_{\mathbb{R}^{d}} d x\left[\frac{\hbar^{2}}{2 m}|\nabla \boldsymbol{\Psi}|^{2}+V(x)|\boldsymbol{\Psi}|^{2}+\frac{1}{2} g_{n}|\boldsymbol{\Psi}|^{4}+\frac{1}{2} g_{s}\left|\boldsymbol{\Psi}^{\dagger} \mathbf{F} \boldsymbol{\Psi}\right|^{2}\right] .
$$

Here, the notation $\boldsymbol{\Psi}^{\dagger}$ stands for $\left(\psi_{1}^{*}, \psi_{0}^{*}, \psi_{-1}^{*}\right), \mathbf{F}=F_{x} \mathbf{e}_{x}+F_{y} \mathbf{e}_{y}+F_{z} \mathbf{e}_{z}$, and $F_{x}, F_{y}, F_{z}$ are the Pauli spinor matrices

$$
F_{x}=\frac{1}{\sqrt{2}}\left(\begin{array}{ccc}
0 & 1 & 0 \\
1 & 0 & 1 \\
0 & 1 & 0
\end{array}\right), \quad F_{y}=\frac{i}{\sqrt{2}}\left(\begin{array}{ccc}
0 & -1 & 0 \\
1 & 0 & -1 \\
0 & 1 & 0
\end{array}\right), \quad F_{z}=\left(\begin{array}{ccc}
1 & 0 & 0 \\
0 & 0 & 0 \\
0 & 0 & -1
\end{array}\right) .
$$

The quantity $\boldsymbol{\Psi}^{\dagger} \mathbf{F} \boldsymbol{\Psi}$ is the expectation (vector) of the spin, whereas $\left|\boldsymbol{\Psi}^{\dagger} \mathbf{F} \boldsymbol{\Psi}\right|^{2}$ standards for the spin-spin interaction energy. More precisely,

$$
\boldsymbol{\Psi}^{\dagger} \mathbf{F} \boldsymbol{\Psi}=\boldsymbol{\Psi}^{\dagger} F_{x} \boldsymbol{\Psi} \mathbf{e}_{x}+\boldsymbol{\Psi}^{\dagger} F_{y} \boldsymbol{\Psi} \mathbf{e}_{y}+\boldsymbol{\Psi}^{\dagger} F_{z} \boldsymbol{\Psi} \mathbf{e}_{z} .
$$

The parameters

$$
g_{n}=\frac{4 \pi \hbar^{2}}{m} \frac{a_{0}+2 a_{2}}{3}, \quad g_{s}=\frac{4 \pi \hbar^{2}}{m} \frac{-a_{0}+a_{2}}{3},
$$

and $a_{0}, a_{2}$ are respectively the scattering lengths corresponding to the total spin zero channel and two channel. The parameter $g_{n}$ characterizes the hyperfine-state independent interaction, while the parameter $g_{s}$ characterizes the spin-exchange interaction.

Due to Feshbach resonance, the s-wave scattering length $a_{0}$ can be tuned over a large range by adjusting the optical dipole trap. Therefore, the parameters $g_{n}$ and $g_{s}$ can be positive or negative. For $g_{n}<0$ (resp. $g_{n}>0$ ), the spinindependent interaction is attractive (resp. repulsive). For $g_{s}<0$ (resp. $g_{s}>0$ ), the spin-exchange interaction is ferromagnetic (resp. anti-ferromagnetic).

This generalized GPE in component form is

$$
\left\{\begin{array}{l}
i \hbar \partial_{t} \psi_{1}=\left(-\frac{\hbar^{2}}{2 m} \nabla^{2}+V(x)+g_{n} n\right) \psi_{1}+g_{s}\left(n_{1}+n_{0}-n_{-1}\right) \psi_{1}+g_{s} \psi_{-1}^{*} \psi_{0}^{2}, \\
i \hbar \partial_{t} \psi_{0}=\left(-\frac{\hbar^{2}}{2 m} \nabla^{2}+V(x)+g_{n} n\right) \psi_{0}+g_{s}\left(n_{1}+n_{-1}\right) \psi_{0}+g_{s} \psi_{1} \psi_{-1} \psi_{0}^{*}, \\
i \hbar \partial_{t} \psi_{-1}=\left(-\frac{\hbar^{2}}{2 m} \nabla^{2}+V(x)+g_{n} n\right) \psi_{-1}+g_{s}\left(n_{-1}+n_{0}-n_{1}\right) \psi_{-1}+g_{s} \psi_{1}^{*} \psi_{0}^{2} .
\end{array}\right.
$$


Here,

$$
n_{j}=\left|\psi_{j}\right|^{2}, \quad j=-1,0,1, \quad \text { and } \quad n=n_{1}+n_{0}+n_{-1} .
$$

From (1.3), the following two integrals are conserved in time:

$$
\begin{aligned}
& \int_{\mathbb{R}^{d}}\left(\left|u_{1}\right|^{2}+\left|u_{2}\right|^{2}+\left|u_{3}\right|^{2}\right)=N, \\
& \int_{\mathbb{R}^{d}}\left(\left|u_{1}\right|^{2}-\left|u_{3}\right|^{2}\right)=M .
\end{aligned}
$$

In this paper, we give a first mathematical study on the ground states associated with (1.3). We consider the simplest case when $V(x) \equiv 0$ and all $\psi_{i}(i=-1,0,1)$ are real. We rename $\psi_{i}$ by $u_{i+2}$. By rescaling, we may assume that $\frac{\hbar^{2}}{2 m}=1$. The energy functional associated with (1.3) is

$$
\begin{aligned}
\mathbb{E}\left(u_{1}, u_{2}, u_{3}\right)= & \frac{1}{2} \int_{\mathbb{R}^{d}} \sum_{j=1}^{3}\left|\nabla u_{j}\right|^{2}+\frac{1}{4} g_{n} \int_{\mathbb{R}^{d}}\left(\sum_{j=1}^{3}\left|u_{j}\right|^{2}\right)^{2} \\
& +\frac{1}{4} g_{s} \int_{\mathbb{R}^{d}}\left[\left(u_{1}^{2}-u_{3}^{2}\right)^{2}+2 u_{2}^{2}\left(u_{1}^{2}+u_{3}^{2}\right)+4 u_{1} u_{3} u_{2}^{2}\right] .
\end{aligned}
$$

It is natural to assume that

$$
N>0, \quad|M|<N .
$$

For given real numbers $(N, M)$, we define

$$
\begin{aligned}
\mathbb{H}_{N, M}= & \left\{u=\left(u_{1}, u_{2}, u_{3}\right) \mid u_{j} \in H^{1}\left(\mathbb{R}^{d}\right), \quad j=1,2,3,\right. \\
& \left.\int_{\mathbb{R}^{d}} \sum_{j=1}^{3}\left|u_{j}\right|^{2}=N, \quad \int_{\mathbb{R}^{d}}\left(\left|u_{1}\right|^{2}-\left|u_{3}\right|^{2}\right)=M\right\} .
\end{aligned}
$$

We consider the minimization problem

$$
E_{0}=\inf \left\{\mathbb{E}(u) \mid u \in \mathbb{H}_{N, M}\right\} .
$$

A solution to (1.8) is called a ground state since it has the smallest energy. A ground state $\left(u_{1}, u_{2}, u_{3}\right)$ is nontrivial if $u_{j} \not \equiv 0$, for $j=1,2,3$.

Our main result in this paper is the following

Theorem 1.1. Let $d=1$ and

$$
g_{n}<g_{s}<0 .
$$

Then a nontrivial ground state exists. Moreover, the ground state $\left(u_{1}, u_{2}, u_{3}\right)$ is positive and strictly decreasing in $|x|$.

Theorem 1.1 is proved via approximation. Namely, we consider a related minimization problem in a bounded interval $I_{k}:=[-k, k]$ and then let $k \rightarrow$ $+\infty$. More precisely, let us define an energy functional on $I_{k}$ : 


$$
\begin{aligned}
\mathbb{E}^{k}\left(u_{1}, u_{2}, u_{3}\right)= & \frac{1}{2} \int_{I_{k}} \sum_{j=1}^{3}\left|\nabla u_{j}\right|^{2}+\frac{1}{4} g_{n} \int_{I_{k}}\left(\sum_{j=1}^{3}\left|u_{j}\right|^{2}\right)^{2} \\
& +\frac{1}{4} g_{s} \int_{I_{k}}\left[\left(u_{1}^{2}-u_{3}^{2}\right)^{2}+2 u_{2}^{2}\left(u_{1}^{2}+u_{3}^{2}\right)+4 u_{1} u_{3} u_{2}^{2}\right]
\end{aligned}
$$

As before, for given real numbers $(N, M)$, we define

$$
\begin{gathered}
\mathbb{H}_{N, M}^{k}=\left\{u=\left(u_{1}, u_{2}, u_{3}\right) \mid u_{j} \in H_{0}^{1}\left(I_{k}\right), j=1,2,3,\right. \\
\left.\int_{I_{k}} \sum_{j=1}^{3}\left|u_{j}\right|^{2}=N, \int_{I_{k}}\left(\left|u_{1}\right|^{2}-\left|u_{3}\right|^{2}\right)=M\right\} .
\end{gathered}
$$

We consider the approximate minimization problem

$$
E_{0}^{k}=\inf \left\{\mathbb{E}^{k}(u) \mid u \in \mathbb{H}_{N, M}^{k}\right\} .
$$

It is easy to see that

$$
E_{0}^{k} \rightarrow E_{0} \quad \text { as } k \rightarrow+\infty
$$

We will prove

Theorem 1.2. Assume that (1.9) holds. Then the minimization problem (1.11) can be attained by some $\mathbf{u}_{k}=\left(u_{1, k}, u_{2, k}, u_{3, k}\right)$ where $u_{j, k}>0$ and strictly decreasing in $|x|$.

The assumption (1.9) is almost necessary. In fact we have

Theorem 1.3. Suppose that $g_{n} \geq 0, g_{s} \geq 0$. Then (1.8) cannot be achieved and hence there is no ground state.

Remarks. (1) Theorem 1.3 says that when $g_{n} \geq 0$ and $g_{s} \geq 0$, both spin-independent interaction and spin-exchange interaction are repulsive, and because there is no trapping potential, the atoms cannot be confined, there is no nontrivial ground state.

(2) When $g_{n}<0$, each hyperfine state is confined to form a spike due to self-attractive interaction. Theorem 1.1 characterize its normalized shape (symmetric about origin and decreasing in $r$ ). When $g_{s}<0$, the spin-exchange interaction is also attractive, the three hyperfine states overlap to each other with peaks at origin. The condition $g_{n}<g_{s}$ should only be a technical condition.

(3) The fact that we can only prove the existence of ground state in the one-dimensional case is a serious restriction due to the Gagliardo-Nirenberg's inequality 3.4. This is also related to the fact that the critical exponent for nonlinear Schrödinger equation is $1+\frac{4}{N}$ which equals 3 when $N=2$.

There are two difficulties in proving Theorem 1.1. First, we need to show that all components of the minimizers are strictly positive. Second, in the onedimensional case, the set of even and strictly decreasing functions in $H^{1}(\mathbb{R})$ is not compact in $L^{2}(\mathbb{R})$. To deal with the first difficulty, we have to use the 
special structure of the energy functional. To overcome the second difficulty, we solve the minimization problem (1.11) first and then show the compactness.

\section{Proof of Theorem 1.3}

From the definition of $\mathbb{E}\left(u_{1}, u_{2}, u_{3}\right)$ we have

$$
\begin{aligned}
\mathbb{E}\left(u_{1}, u_{2}, u_{3}\right)= & \frac{1}{2} \int_{\mathbb{R}}\left(\left|u_{1}^{\prime}\right|^{2}+\left|u_{2}^{\prime}\right|^{2}+\left|u_{3}^{\prime}\right|^{2}\right)+\frac{1}{4} g_{n} \int_{\mathbb{R}}\left(u_{1}^{2}+u_{2}^{2}+u_{3}^{2}\right)^{2} \\
& +\frac{1}{4} g_{s} \int_{\mathbb{R}}\left[2 u_{2}^{2}\left(u_{1}+u_{3}\right)^{2}+\left(u_{1}^{2}-u_{3}^{2}\right)^{2}\right] .
\end{aligned}
$$

If $g_{n} \geq 0, g_{s} \geq 0$ we always have $E_{0} \geq 0$. We will show that $E_{0}=0$ if $g_{n} \geq 0, g_{s} \geq 0$. Suppose that $\left(u_{1}, u_{2}, u_{3}\right)$ such that $u_{j} \in H^{1}(\mathbb{R})$ satisfies

$$
\int_{\mathbb{R}} \sum_{j=1}^{3}\left|u_{j}\right|^{2}=N, \quad \int_{\mathbb{R}}\left(\left|u_{1}\right|^{2}-\left|u_{3}\right|^{2}\right)=M .
$$

Set $v_{j}(x)=\rho^{\frac{1}{2}} u_{j}(\rho x)$ for $j=1,2,3$. Then for any $\rho>0,\left(v_{1}, v_{2}, v_{3}\right)$ also satisfies

$$
\int_{\mathbb{R}} \sum_{j=1}^{3}\left|v_{j}\right|^{2}=N, \quad \int_{\mathbb{R}}\left(\left|v_{1}\right|^{2}-\left|v_{3}\right|^{2}\right)=M .
$$

On the other hand, we have

$$
\begin{aligned}
\mathbb{E}\left(v_{1}, v_{2}, v_{3}\right)= & \frac{1}{2} \rho^{2} \int_{\mathbb{R}}\left(\left|u_{1}^{\prime}\right|^{2}+\left|u_{2}^{\prime}\right|^{2}+\left|u_{3}^{\prime}\right|^{2}\right)+\frac{1}{4} \rho g_{n} \int_{\mathbb{R}}\left(u_{1}^{2}+u_{2}^{2}+u_{3}^{2}\right)^{2} \\
& +\frac{1}{4} \rho g_{s} \int_{\mathbb{R}}\left[2 u_{2}^{2}\left(u_{1}+u_{3}\right)^{2}+\left(u_{1}^{2}-u_{3}^{2}\right)^{2}\right] .
\end{aligned}
$$

Taking $\rho \rightarrow 0$ we have $E_{0}=0$. Therefore $E_{0}$ can be achieved only by $(0,0,0) \notin$ $\mathbb{H}_{N, M}$. This proves Theorem 1.3.

A corollary of the above proof is the following

Corollary 2.1. If $g_{n}<0, g_{s}<0$ then $E_{0}<0$.

In fact, this follows directly from (2.2), by taking $\rho$ small enough.

\section{Proof of Theorem 1.2}

We now consider the minimization problem (1.11) and prove Theorem 1.2.

From the definition of $\mathbb{E}^{k}\left(u_{1}, u_{2}, u_{3}\right)$, we have

$$
\begin{aligned}
\mathbb{E}^{k}\left(u_{1}, u_{2}, u_{3}\right) & \\
= & \frac{1}{2} \int_{I_{k}}\left(\left|u_{1}^{\prime}\right|^{2}+\left|u_{2}^{\prime}\right|^{2}+\left|u_{3}^{\prime}\right|^{2}\right)+\frac{1}{4}\left(g_{n}+g_{s}\right) \int_{I_{k}}\left(u_{1}^{4}+u_{3}^{4}\right)+\frac{1}{4} g_{n} \int_{I_{k}} u_{2}^{4} \\
& +\frac{1}{4}\left(g_{n}+g_{s}\right) \int_{I_{k}}\left(2 u_{2}^{2}\left(u_{1}^{2}+u_{3}^{2}\right)\right)+\frac{1}{4}\left(g_{n}-g_{s}\right) \int_{I_{k}} 2 u_{1}^{2} u_{3}^{2} \\
& +\frac{1}{4} g_{s} \int_{I_{k}}\left(4 u_{1} u_{3} u_{2}^{2}\right) .
\end{aligned}
$$


Let $\mathbf{u}^{\ell}=\left(u_{1}^{\ell}, u_{2}^{\ell}, u_{3}^{\ell}\right)$ be a minimizing sequence of (1.11). We can always assume that each component $u_{j}^{\ell}$ is non-negative, since it is easy to see that

$$
\mathbb{E}^{k}\left(\left|u_{1}^{\ell}\right|,\left|u_{2}^{\ell}\right|,\left|u_{3}^{\ell}\right|\right) \leq \mathbb{E}^{k}\left(u_{1}^{\ell}, u_{2}^{\ell}, u_{3}^{\ell}\right)
$$

and $\left(\left|u_{1}^{\ell}\right|,\left|u_{2}^{\ell}\right|,\left|u_{3}^{\ell}\right|\right) \in \mathbb{H}_{N, M}^{k}$. Hence we can replace $\left(u_{1}^{\ell}, u_{2}^{\ell}, u_{3}^{\ell}\right)$ by $\left(\left|u_{1}^{\ell}\right|,\left|u_{2}^{\ell}\right|\right.$, $\left.\left|u_{3}^{\ell}\right|\right)$.

For $v \in H^{1}(\mathbb{R}), v \geq 0$, let us denote its Schwarz symmetrization by $v^{*}$. Then we have (see Theorem 3.2 of Lieb and Loss [14])

$$
\left\{\begin{array}{l}
\int_{\mathbb{R}}\left|u_{j}^{\prime}\right|^{2} \geq \int_{\mathbb{R}}\left|\left(u_{j}^{*}\right)^{\prime}\right|^{2}, \quad j=1,2,3, \\
\int_{\mathbb{R}} u_{j}^{4}=\int_{\mathbb{R}}\left(u_{j}^{*}\right)^{4}, \quad j=1,2,3, \\
\int_{\mathbb{R}} u_{j}^{2} u_{k}^{2} \leq \int_{\mathbb{R}}\left(u_{j}^{*}\right)^{2}\left(u_{k}^{*}\right)^{2}, \quad j, k=1,2,3, \\
\int_{\mathbb{R}} u_{1} u_{3} u_{2}^{2} \leq \int_{\mathbb{R}} u_{1}^{*} u_{3}^{*}\left(u_{2}^{*}\right)^{2}
\end{array}\right.
$$

which implies (by (3.1)) that

$$
\mathbb{E}^{k}\left(\left(u_{1}^{\ell}\right)^{*},\left(u_{2}^{\ell}\right)^{*},\left(u_{3}^{\ell}\right)^{*}\right) \leq \mathbb{E}^{k}\left(u_{1}^{\ell}, u_{2}^{\ell}, u_{3}^{\ell}\right)
$$

and $\left(\left(u_{1}^{\ell}\right)^{*},\left(u_{2}^{\ell}\right)^{*},\left(u_{3}^{\ell}\right)^{*}\right) \in \mathbb{H}_{N, M}^{k}$.

As a consequence, we can now assume that $u_{j}^{\ell}$ are nonnegative, even and non-increasing in $I_{k}$.

Next we show that minimizing sequence is uniformly bounded in $H_{0}^{1}\left(I_{k}\right)$ for $k \geq 1$. Recall that

$$
\mathbb{E}^{k}\left(u_{1}^{\ell}, u_{2}^{\ell}, u_{3}^{\ell}\right) \rightarrow E_{0}^{k}(l \rightarrow \infty), \quad \int_{I_{k}}\left(\left(u_{1}^{\ell}\right)^{2}+\left(u_{2}^{\ell}\right)^{2}+\left(u_{3}^{\ell}\right)^{2}\right)=N .
$$

By Gagliardo-Nirenberg inequality [25]

$$
\int_{\mathbb{R}}|u|^{4} \leq C\left(\int_{\mathbb{R}}\left|u^{\prime}\right|^{2}\right)^{\frac{1}{2}}\left(\int_{\mathbb{R}}|u|^{2}\right)^{\frac{3}{2}}, \quad \text { for all } u \in H^{1}(\mathbb{R}),
$$

we have for any $\epsilon>0$, there exists $C(\epsilon)>0$ such that

$$
\begin{aligned}
\int_{I_{k}}|u|^{4} & \leq C \epsilon \int_{I_{k}}\left|u^{\prime}\right|^{2}+C(\epsilon)\left(\int_{I_{k}}|u|^{2}\right)^{3} \\
\int_{I_{k}} u^{2} v^{2} & \leq C\left(\int_{I_{k}} u^{4}\right)^{\frac{1}{2}}\left(\int_{I_{k}} v^{4}\right)^{\frac{1}{2}} \leq C \int_{I_{k}} u^{4}+C \int_{I_{k}} v^{4} \\
& \leq C \epsilon\left(\int_{I_{k}}\left|u^{\prime}\right|^{2}+\int_{I_{k}}\left|v^{\prime}\right|^{2}\right)+C(\epsilon)\left(\left(\int_{I_{k}}|u|^{2}\right)^{3}+\left(\int_{I_{k}}|v|^{2}\right)^{3}\right),
\end{aligned}
$$

and

$$
\begin{aligned}
\left|\int_{I_{k}} u^{2} v w\right| \leq & C\left(\int_{I_{k}} u^{4}\right)^{\frac{1}{2}}\left(\int_{I_{k}} v^{2} w^{2}\right)^{\frac{1}{2}} \leq C \int_{I_{k}} u^{4}+C \int_{I_{k}} v^{2} w^{2} \\
\leq & C \epsilon\left(\int_{I_{k}}\left|u^{\prime}\right|^{2}+\left|v^{\prime}\right|^{2}+\left|w^{\prime}\right|^{2}\right) \\
& +C(\epsilon)\left(\left(\int_{I_{k}}|u|^{2}\right)^{3}+\left(\int_{I_{k}}|v|^{2}\right)^{3}+\left(\int_{I_{k}} w^{2}\right)^{3}\right) .
\end{aligned}
$$


Hence, making use of the above inequalities, we get

$$
\begin{aligned}
\frac{1}{2} \int_{I_{k}} \sum_{j=1}^{3}\left|\left(u_{j}^{\ell}\right)^{\prime}\right|^{2}= & E_{0}-\frac{1}{4} g_{n} \int_{I_{k}}\left(\left(u_{1}^{\ell}\right)^{2}+\left(u_{2}^{\ell}\right)^{2}+\left(u_{3}^{\ell}\right)^{2}\right)^{2} \\
& -\frac{1}{4} g_{s} \int_{I_{k}}\left[2\left(u_{2}^{\ell}\right)^{2}\left(u_{1}^{\ell}+u_{3}^{\ell}\right)^{2}+\left(\left(u_{1}^{\ell}\right)^{2}-\left(u_{3}^{\ell}\right)^{2}\right)^{2}\right]+o_{\ell}(1) \\
\leq & E_{0}-\frac{C}{4}\left(g_{n}+g_{s}\right) \epsilon\left(\int_{I_{k}}\left[\left|\left(u_{1}^{\ell}\right)^{\prime}\right|^{2}+\left|\left(u_{2}^{\ell}\right)^{\prime}\right|^{2}+\left|\left(u_{3}^{\ell}\right)^{\prime}\right|^{2}\right]\right) \\
& +C(\epsilon) N^{3} .
\end{aligned}
$$

where $o_{\ell}(1) \rightarrow 0$ as $\ell \rightarrow+\infty$.

Choosing $\epsilon$ sufficiently small, we see

$$
\int_{I_{k}}\left[\sum_{j=1}^{3}\left(\left|\left(u_{j}^{\ell}\right)^{\prime}\right|^{2}+\left(u_{j}^{\ell}\right)^{2}\right)\right]<C
$$

which implies that by Sobolev embedding

$$
\left|u_{j}^{\ell}\right|_{L^{\infty}}<C, \quad j=1,2,3, \quad \ell=1,2, \ldots .
$$

(Let us remark that the constant $C$ here is independent of $k \geq 1$.)

Thus, $\left\{u_{j}^{\ell}\right\}$ cannot blow up in $\mathbb{R}, j=1,2,3$.

Therefore from $\mathbf{u}^{\ell}(x)$ we can obtain the existence of minimizer $\left(u_{1, k}, u_{2, k}\right.$, $\left.u_{3, k}\right)$ by applying compactness of embedding of subspace of $H_{0}^{1}\left(I_{k}\right)$ consisting of even functions into $L^{4}\left(I_{k}\right)$. We can also assume that $u_{j, k} \geq 0$ for $j=$ $1,2,3$ and at least one component is not identically 0. Furthermore, there are Lagrange multipliers $\lambda^{k}, \mu^{k}$ such that $\left(u_{1, k}, u_{2, k}, u_{3, k}\right)$ satisfies the EulerLagrange equation

$$
\begin{cases}u_{1}^{\prime \prime}-\left(g_{n}+g_{s}\right) u_{1}^{3}-\left[\left(g_{n}+g_{s}\right) u_{2}^{2}+\left(g_{n}-g_{s}\right) u_{3}^{2}\right] u_{1}-g_{s} u_{2}^{2} u_{3}=\left(\lambda^{k}+\mu^{k}\right) u_{1}, & \text { in } I_{k} \\ u_{2}^{\prime \prime}-g_{n} u_{2}^{3}-\left[\left(g_{n}+g_{s}\right) u_{1}^{2}+\left(g_{n}+g_{s}\right) u_{3}^{2}\right] u_{2}-2 g_{s} u_{1} u_{2} u_{3}=\lambda^{k} u_{2}, & \text { in } I_{k} \\ u_{3}^{\prime \prime}-\left(g_{n}+g_{s}\right) u_{3}^{3}-\left[\left(g_{n}+g_{s}\right) u_{2}^{2}+\left(g_{n}-g_{s}\right) u_{1}^{2}\right] u_{3}-g_{s} u_{1} u_{2}^{2}=\left(\lambda^{k}-\mu^{k}\right) u_{3}, & \text { in } I_{k} \\ u_{j}>0 \text { in }(-k, k), u_{j}( \pm k)=0, \quad j=1,2,3 . & \end{cases}
$$

Suppose that $\left(u_{1, k}, u_{2, k}, u_{3, k}\right)$ is a minimizer of (1.11). It remains to show that $u_{j, k} \neq \equiv 0$, for $j=1,2,3$. This will be done by two claims.

Claim 1. $u_{2, k}>0$.

We argue by contradiction. Suppose that $u_{2, k} \geq 0$ and $u_{2, k}\left(x_{0}\right)=0$. Then by the Maximum Principle, $u_{2, k} \equiv 0$, and hence $\left(u_{1, k}, u_{3, k}\right)$ is a solution of

$$
\begin{cases}u_{1}^{\prime \prime}-\left(g_{n}+g_{s}\right) u_{1}^{3}-\left(g_{n}-g_{s}\right) u_{3}^{2} u_{1}=\left(\lambda^{k}+\mu^{k}\right) u_{1}, & \text { in } I_{k}, \\ u_{3}^{\prime \prime}-\left(g_{n}+g_{s}\right) u_{3}^{3}-\left(g_{n}-g_{s}\right) u_{1}^{2} u_{3}=\left(\lambda^{k}-\mu^{k}\right) u_{3}, & \text { in } I_{k}\end{cases}
$$

satisfying the constraint

$$
\int_{I_{k}} u_{1, k}^{2}=\frac{N+M}{2}, \quad \int_{I_{k}} u_{3, k}^{2}=\frac{N-M}{2} .
$$


Let $\left(\varphi_{1}, \varphi_{2}, \varphi_{3}\right)$ be such that

$$
\varphi_{1}=-u_{1, k}, \quad \varphi_{2, k}=u_{1, k}+\eta u_{3, k}, \quad \varphi_{3, k}=-u_{3, k}
$$

where $\eta=\left(\frac{N+M}{N-M}\right)^{\frac{1}{2}}$, and let $\varepsilon_{2}>0$ be a fixed small number.

Then by implicit function theorem, we can find $\varepsilon_{1}>0, \varphi_{3}>0$ small such that

$$
\begin{aligned}
& \int_{I_{k}}\left(\left|u_{1, k}+\varepsilon_{1} \varphi_{1}\right|^{2}+\left|\varepsilon_{2} \varphi_{2}\right|^{2}+\left|u_{3, k}+\varepsilon_{3} \varphi_{3}\right|^{2}\right)=N \\
& \int_{I_{k}}\left(\left|u_{1, k}+\varepsilon_{1} \varphi_{1}\right|^{2}-\left|u_{3, k}+\varepsilon_{3} \varphi_{3}\right|^{2}\right)=M
\end{aligned}
$$

In fact, (3.11)-(3.12) are equivalent to

$$
\begin{aligned}
& 2 \varepsilon_{1} \int_{I_{k}} u_{1, k} \varphi_{1}+\varepsilon_{1}^{2} \int_{I_{k}}\left|\varphi_{1}\right|^{2}+2 \varepsilon_{3} \int_{I_{k}} u_{3, k} \varphi_{3}+\varepsilon_{3}^{2} \int_{I_{k}}\left|\varphi_{3}\right|^{2} \\
& \quad+\varepsilon_{2}^{2} \int_{I_{k}}\left|\varphi_{2}\right|^{2}=0, \\
& 2 \varepsilon_{1} \int_{I_{k}} u_{1, k} \varphi_{1}+\varepsilon_{1}^{2} \int_{I_{k}}\left|\varphi_{1}\right|^{2}=2 \varepsilon_{3} \int_{I_{k}} u_{3, k} \varphi_{3}+\varepsilon_{3}^{2} \int_{I_{k}}\left|\varphi_{3}\right|^{2},
\end{aligned}
$$

which are equivalent to

$$
\begin{aligned}
& \varepsilon_{1} \int_{I_{k}} u_{1, k} \varphi_{1}-\varepsilon_{3} \int_{I_{k}} u_{3, k} \varphi_{3}=O\left(\varepsilon_{1}^{2}+\varepsilon_{3}^{2}\right), \\
& \varepsilon_{1} \int_{I_{k}} u_{1, k} \varphi_{1}+\varepsilon_{3} \int_{I_{k}} u_{3, k} \varphi_{3}=-\frac{1}{2} \varepsilon_{2}^{2} \int_{I_{k}}\left|\varphi_{2}\right|^{2}+O\left(\varepsilon_{1}^{2}+\varepsilon_{3}^{2}\right) .
\end{aligned}
$$

By implicit function theorem, we can find $\varepsilon_{1}=O\left(\epsilon_{2}^{2}\right), \varepsilon_{3}=O\left(\varepsilon_{2}^{2}\right)$ satisfying (3.15)-(3.16).

Applying the above equality to the expression in $\mathbb{E}^{k}\left(u_{1}, u_{2}, u_{3}\right)$, we obtain

$$
\begin{aligned}
& \mathbb{E}^{k}\left(u_{1}, u_{2}, u_{3}\right) \\
&=\varepsilon_{1} \int_{I_{k}}\left(\left(u_{1, k}\right)^{\prime} \varphi_{1}^{\prime}+\left(g_{n}+g_{s}\right)\left(u_{1, k}\right)^{3} \varphi_{1}+\left(g_{n}-g_{s}\right)\left(u_{3, k}\right)^{2} u_{1, k} \varphi_{1}\right) \\
&+\varepsilon_{3} \int_{I_{k}}\left(\left(u_{3, k}\right)^{\prime} \varphi_{3}^{\prime}+\left(g_{n}+g_{s}\right)\left(u_{3, k}\right)^{3} \varphi_{3}+\left(g_{n}-g_{s}\right)\left(u_{1, k}\right)^{2} u_{3, k} \varphi_{3}\right) \\
&+\varepsilon_{2}^{2}\left[\frac{1}{2} \int_{I_{k}}\left(\left|\varphi_{2}^{\prime}\right|^{2}+\left(g_{n}+g_{s}\right)\left(\left(u_{1, k}\right)^{2}+\left(u_{3, k}\right)^{2}\right) \varphi_{2}^{2}\right)+g_{s} \int_{I_{k}} u_{1, k} u_{3, k}\left|\varphi_{2}\right|^{2}\right] \\
&+\mathbb{E}^{k}\left(u_{1, k}, 0, u_{3, k}\right)+o\left(\varepsilon_{1}^{2}+\varepsilon_{3}^{2}\right) \\
&=-\varepsilon_{1}\left(\lambda^{k}+\mu^{k}\right) \int_{I_{k}} u_{1, k} \varphi_{1}-\varepsilon_{3}\left(\lambda^{k}-\mu^{k}\right) \int_{I_{k}} u_{3, k} \varphi_{3} \\
&+\varepsilon_{2}^{2}\left[\frac{1}{2} \int_{I_{k}}\left(\left|\varphi_{2}^{\prime}\right|^{2}+\left(g_{n}+g_{s}\right)\left(\left(u_{1, k}\right)^{2}+\left(u_{3, k}\right)^{2}\right) \varphi_{2}^{2}\right)+g_{s} \int_{I_{k}} u_{1, k} u_{3, k}\left|\varphi_{2}\right|^{2}\right] \\
&+\mathbb{E}^{k}\left(u_{1, k}, 0, u_{3, k}\right)+o\left(\varepsilon_{1}^{2}+\varepsilon_{3}^{2}\right)
\end{aligned}
$$




$$
\begin{aligned}
= & \frac{1}{2} \varepsilon_{2}^{2} \int_{I_{k}}\left[\left|\varphi_{2}^{\prime}\right|^{2}+\lambda^{k}\left|\varphi_{2}\right|^{2}+\left(g_{n}+g_{s}\right)\left(\left(u_{1, k}\right)^{2}+\left(u_{3, k}\right)^{2}\right) \varphi_{2}^{2}+2 g_{s} u_{1, k} u_{3, k}\left|\varphi_{2}\right|^{2}\right] \\
& +\mathbb{E}^{k}\left(u_{1, k}, 0, u_{3, k}\right)+o\left(\varepsilon_{1}^{2}+\varepsilon_{3}^{2}\right) .
\end{aligned}
$$

We claim that if $g_{s}<0$ then

$$
\int_{I_{k}}\left[\left|\varphi_{2}^{\prime}\right|^{2}+\lambda^{k} \varphi_{2}^{2}+\left(g_{n}+g_{s}\right)\left(\left(u_{1, k}\right)^{2}+\left(u_{3, k}\right)^{2}\right) \varphi_{2}^{2}\right]+2 g_{s} \int_{I_{k}} u_{1, k} u_{3, k} \varphi_{2}^{2}<0 \text {. }
$$

Indeed, from (3.8) and the choice of $\eta$ we have

$$
\begin{aligned}
& \int_{I_{k}}\left(\left|\left(u_{1, k}\right)^{\prime}\right|^{2}+\eta^{2}\left|\left(u_{3, k}\right)^{\prime}\right|^{2}+\left(g_{n}+g_{s}\right)\left(\left(u_{1, k}\right)^{4}+\eta^{2}\left(u_{3, k}\right)^{4}\right)\right. \\
& \left.\quad+2\left(g_{n}-g_{s}\right)\left(1+\eta^{2}\right)\left(u_{1, k}\right)^{2}\left(u_{3, k}\right)^{2}\right) \\
& \quad+\lambda^{k} \int_{I_{k}}\left(\left(u_{1, k}\right)^{2}+\eta^{2}\left(u_{3, k}\right)^{2}\right)=0, \\
& \int_{I_{k}}\left[\left(u_{1, k}\right)^{\prime}\left(u_{3, k}\right)^{\prime}+\lambda^{k} u_{1, k} u_{3, k}+g_{n}\left(\left(u_{1, k}\right)^{3} u_{3, k}+\left(u_{3, k}\right)^{3} u_{3, k}\right)\right]=0 .
\end{aligned}
$$

Using (3.19), (3.20) we have

$$
\begin{aligned}
& \int_{I_{k}}\left[\left|\left(u_{1, k}+\eta u_{3, k}\right)^{\prime}\right|^{2}+\lambda^{k}\left(u_{1, k}+\eta u_{3, k}\right)^{2}+\left(g_{n}+g_{s}\right)\left(\left(u_{1, k}\right)^{4}+\eta^{2}\left(u_{3, k}\right)^{4}\right)\right] \\
& \quad+2 \eta g_{n} \int_{I_{k}}\left(\left(u_{1, k}\right)^{3} u_{3, k}+\left(u_{3, k}\right)^{3} u_{1, k}\right)+\left(g_{n}-g_{s}\right)\left(1+\eta^{2}\right) \int_{I_{k}}\left(u_{1, k}\right)^{2}\left(u_{3, k}\right)^{2}=0 .
\end{aligned}
$$

So, since $g_{s}<0$ and $u_{1, k} u_{3, k}$ is not identically zero, we get

$$
\begin{aligned}
& \int_{I_{k}}\left[\left|\left(u_{1, k}+\eta u_{3, k}\right)^{\prime}\right|^{2}+\lambda^{k}\left(u_{1, k}+\eta u_{3, k}\right)^{2}\right. \\
& \left.\quad+\left(g_{n}+g_{s}\right)\left(\left(u_{1, k}\right)^{2}+\left(u_{3, k}\right)^{2}\right)\left(u_{1, k}+\eta u_{3, k}\right)^{2}\right]+2 g_{s} \int_{I_{k}} u_{1, k} u_{3, k}\left(u_{1, k}+\eta u_{3, k}\right)^{2} \\
& \quad=2(1+\eta) g_{s} \int_{I_{k}} u_{1, k} u_{3, k}\left((1+\eta) u_{1, k} u_{3, k}+\left(u_{1, k}\right)^{2}+\eta\left(u_{3, k}\right)^{2}\right)<0 .
\end{aligned}
$$

Since $\varepsilon_{1}=O\left(\varepsilon_{2}^{2}\right), \varepsilon_{3}=O\left(\varepsilon_{2}^{2}\right)$, from (3.17) and (3.22), we conclude that we can choose an element $\mathbf{u} \in \mathbb{H}_{N, M}^{k}$ such that $\mathbb{E}^{k}(\mathbf{u})<E_{0}$. This is a contradiction to the minimality of $\left(u_{1, k}, u_{2, k}, u_{3, k}\right)$.

Therefore $u_{2, k}$ is a positive function.

Claim 2. $u_{1, k}>0, u_{3, k}>0$.

Suppose that $u_{1, k} \geq 0$ and $u_{1, k}\left(x_{0}\right)=0$. By the Maximum Principle, $u_{1, k}(x) \equiv 0$. By the equation satisfied by $u_{1, k}$ we obtain $g_{s}\left(u_{2, k}\right)^{2} u_{3, k} \equiv 0$. Therefore either $u_{2, k} \equiv 0$ or $u_{3, k} \equiv 0$. By Claim $1, u_{2, k}>0$. Therefore the 
only possibility is that $u_{3, k} \equiv 0$. In this case, $M=0$ and $\int_{I_{k}}\left(u_{2, k}\right)^{2}=N$ and $u_{2, k}$ is a positive solution of

$$
u^{\prime \prime}-g_{n} u^{3}=\lambda^{k} u \text { in } I_{k} .
$$

Let $\left(u_{1}, u_{2}, u_{3}\right)=\left(\varepsilon_{1} \varphi_{1}, u_{2, k}+\varepsilon_{2} \varphi_{2}, \varepsilon_{3} \varphi_{3}\right)$ where $\varepsilon_{1}=\varepsilon_{3}=\varepsilon, \varphi_{1}=\varphi_{3}=$ $\varphi=u_{2, k}$ and $\varphi_{2}=-u_{2, k}$. Then for $\varepsilon>0$ sufficiently small, we can find $\varepsilon_{2}>0$ small such that

$$
\begin{aligned}
& \varepsilon_{1}^{2} \int_{I_{k}}\left|\varphi_{1}\right|^{2}=\varepsilon_{3}^{2} \int_{I_{k}}\left|\varphi_{3}\right|^{2}, \\
& \varepsilon_{1}^{2} \int_{I_{k}}\left|\varphi_{1}\right|^{2}+\varepsilon_{3}^{2} \int_{I_{k}}\left|\varphi_{3}\right|^{2}+\varepsilon_{2}^{2} \int_{I_{k}}\left|\varphi_{2}\right|^{2}+2 \varepsilon_{2} \int_{I_{k}} u_{2, k} \varphi_{2}=0 .
\end{aligned}
$$

From (3.25), we derive that $\varepsilon_{2}=O\left(\varepsilon^{2}\right)$.

Since $u_{2, k}$ satisfies (3.23) we have

$$
\int_{I_{k}}\left(\left(u_{2, k}\right)^{\prime} \varphi_{2}^{\prime}+\lambda^{k} u_{2, k} \varphi_{2}+g_{n}\left(u_{2, k}\right)^{3} \varphi_{2}\right)=0 .
$$

From (3.24), (3.25) and (3.26) we have

$$
\begin{aligned}
& \varepsilon_{2} \int_{I_{k}}\left(\left(u_{2, k}\right)^{\prime} \varphi_{2}^{\prime}+g_{n}\left(u_{2, k}\right)^{3} \varphi_{2}\right) \\
& \quad=\frac{1}{2} \lambda^{k}\left(\varepsilon_{1}^{2} \int_{I_{k}}\left|\varphi_{1}\right|^{2}+\varepsilon_{2}^{2} \int_{I_{k}}\left|\varphi_{2}\right|^{2}+\varepsilon_{3}^{2} \int_{I_{k}}\left|\varphi_{3}\right|^{2}\right) .
\end{aligned}
$$

Using (3.27) we get

$$
\begin{aligned}
\mathbb{E}^{k}\left(u_{1}, u_{2}, u_{3}\right)= & \mathbb{E}^{k}\left(\varepsilon_{1} \varphi_{1}, u_{2, k}+\varepsilon_{2} \varphi_{2}, \varepsilon_{3} \varphi_{3}\right) \\
= & \frac{1}{2} \varepsilon_{1}^{2} \int_{I_{k}}\left|\varphi_{1}^{\prime}\right|^{2}+\frac{1}{4}\left(g_{n}+g_{s}\right) \varepsilon_{1}^{4} \int_{I_{k}}\left|\varphi_{1}\right|^{4} \\
& +\frac{1}{2} \varepsilon_{3}^{2} \int_{I_{k}}\left(\left|\varphi_{3}^{\prime}\right|^{2}+\frac{1}{4}\left(g_{n}+g_{s}\right) \varepsilon_{3}^{4} \int_{I_{k}}\left|\varphi_{3}\right|^{4}\right. \\
& +\frac{1}{2} \int_{I_{k}}\left|\left(u_{2, k}+\varepsilon_{2} \varphi_{2}\right)^{\prime}\right|^{2}+\frac{1}{4} g_{n} \int_{I_{k}}\left|u_{2, k}+\varepsilon_{2} \varphi_{2}\right|^{4} \\
& +\frac{1}{2}\left(g_{n}+g_{s}\right) \int_{I_{k}}\left[\left(\varepsilon_{1}^{2} \varphi_{1}^{2}+\varepsilon_{3}^{2} \varphi_{3}^{2}\right)\left(u_{2, k}+\varepsilon_{2} \varphi_{2}\right)^{2}\right] \\
& +\frac{1}{2}\left(g_{n}-g_{s}\right) \int_{I_{k}} \varepsilon_{1}^{2} \varepsilon_{3}^{2} \varphi_{1}^{2} \varphi_{3}^{2}+g_{s} \int_{R} \varepsilon_{1} \varepsilon_{3} \varphi_{1} \varphi_{3}\left(u_{2, k}+\varepsilon_{2} \varphi_{2}\right)^{2} \\
= & \varepsilon_{1}^{2}\left(\frac{1}{2} \int_{I_{k}}\left|\varphi_{1}^{\prime}\right|^{2}+\frac{1}{2}\left(g_{n}+g_{s}\right) \int_{R}\left|u_{2, k} \varphi_{1}\right|^{2}\right) \\
& +\varepsilon_{3}^{2}\left(\frac{1}{2} \int_{I_{k}}\left|\varphi_{3}^{\prime}\right|^{2}+\frac{1}{2}\left(g_{n}+g_{s}\right) \int_{I_{k}}\left|u_{2, k} \varphi_{3}\right|^{2}\right) \\
& +g_{s} \varepsilon_{1} \varepsilon_{3} \int_{I_{k}}\left|u_{2, k}+\varepsilon_{2} \varphi_{2}\right|^{2} \varphi_{1} \varphi_{3} \\
& +\varepsilon_{2}\left[\int_{I_{k}}\left(u_{2, k}\right)^{\prime} \varphi_{2}^{\prime}+g_{n} \int_{I_{k}}\left(u_{2, k}\right)^{3} \varphi_{2}\right]
\end{aligned}
$$




$$
\begin{aligned}
& +\varepsilon_{2}^{2}\left[\frac{1}{2} \int_{I_{k}}\left(\left|\varphi_{2}^{\prime}\right|^{2}+\frac{3}{2} g_{n}\left(u_{2, k}\right)^{2} \varphi_{2}^{2}\right]\right. \\
& +\mathbb{E}^{k}\left(0, u_{2, k}, 0\right)+o\left(\varepsilon_{1}^{2}+\varepsilon_{3}^{2}\right) \\
= & \varepsilon_{1}^{2}\left(\frac{1}{2} \int_{I_{k}}\left(\left|\varphi_{1}^{\prime}\right|^{2}+\lambda^{k}\left|\varphi_{1}\right|^{2}\right)+\frac{1}{2}\left(g_{n}+g_{s}\right) \int_{I_{k}}\left|u_{2, k} \varphi_{1}\right|^{2}\right) \\
& +\varepsilon_{3}^{2}\left(\frac{1}{2} \int_{I_{k}}\left(\left|\varphi_{3}^{\prime}\right|^{2}+\lambda^{k}\left|\varphi_{2}\right|^{2}\right)+\frac{1}{2}\left(g_{n}+g_{s}\right) \int_{I_{k}}\left|u_{2, k} \varphi_{3}\right|^{2}\right) \\
& +g_{s} \varepsilon_{1} \varepsilon_{3} \int_{I_{k}}\left|u_{2, k}\right|^{2} \varphi_{1} \varphi_{3} \\
& +\mathbb{E}^{k}\left(0, u_{2, k}, 0\right)+o\left(\varepsilon_{1}^{2}+\varepsilon_{3}^{2}\right),
\end{aligned}
$$

where we have used the fact that $\varepsilon_{2}=O\left(\varepsilon_{1}^{2}+\varepsilon_{3}^{2}\right)$.

By the choices of $\varepsilon_{j}, \varphi_{j}$, we obtain

$$
\left\{\begin{array}{l}
\varepsilon_{1}^{2}\left(\frac{1}{2} \int_{I_{k}}\left(\left|\varphi_{1}^{\prime}\right|^{2}+\lambda^{k}\left|\varphi_{1}\right|^{2}\right)+\frac{1}{2}\left(g_{n}+g_{s}\right) \int_{I_{k}}\left|u_{2, k} \varphi_{1}\right|^{2}\right) \\
+\varepsilon_{3}^{2}\left(\frac{1}{2} \int_{I_{k}}\left(\left|\varphi_{3}^{\prime}\right|^{2}+\lambda^{k}\left|\varphi_{3}\right|^{2}\right)+\frac{1}{2}\left(g_{n}+g_{s}\right) \int_{I_{k}}\left|u_{2, k} \varphi_{3}\right|^{2}\right) \\
+g_{s} \varepsilon_{1} \varepsilon_{3} \int_{I_{k}}\left|u_{2, k}\right|^{2} \varphi_{1} \varphi_{3} \\
=\varepsilon^{2}\left(\int_{I_{k}}\left(\left|\varphi^{\prime}\right|^{2}+\lambda^{k}|\varphi|^{2}\right)+\left(g_{n}+2 g_{s}\right) \int_{I_{k}}\left|u_{2, k} \varphi\right|^{2}\right)<0
\end{array}\right.
$$

Thus we conclude that

$$
\mathbb{E}^{k}\left(\varepsilon_{1} u_{2, k},\left(1+\varepsilon_{2}\right) u_{2, k}, \varepsilon_{1} u_{2, k}\right)<\mathbb{E}^{k}\left(0, u_{2, k}, 0\right),
$$

which is a contradiction to the minimality of $\left(u_{1, k}, u_{2, k}, u_{3, k}\right)$.

Therefore, $u_{1, k} \not \equiv 0$. Similarly we can show that $u_{3, k} \not \equiv 0$. Thus we have completed the proof of Theorem 1.2.

\section{Proof of Theorem 1.1}

From Sect. 3, for each $k \geq 1$, we obtain a minimizer to the minimization problem (1.11) which satisfies the following Euler-Lagrange equation

$$
\begin{cases}u_{1, k}^{\prime \prime}-\left(g_{n}+g_{s}\right) u_{1, k}^{3}-\left[\left(g_{n}+g_{s}\right) u_{2, k}^{2}+\left(g_{n}-g_{s}\right) u_{3, k}^{2}\right] u_{1, k} & \text { in }(-k, k), \\ -g_{s} u_{2, k}^{2} u_{3, k}=\left(\lambda^{k}+\mu^{k}\right) u_{1, k} & \\ u_{2, k}^{\prime \prime}-g_{n} u_{2, k}^{3}-\left[\left(g_{n}+g_{s}\right) u_{1, k}^{2}+\left(g_{n}+g_{s}\right) u_{3, k}^{2}\right] u_{2, k} & \text { in }(-k, k), u_{3, k}^{\prime \prime} \\ -2 g_{s} u_{1, k} u_{2, k} u_{3, k}=\lambda^{k} u_{2, k} & \\ -\left(g_{n}+g_{s}\right) u_{3, k}^{3}-\left[\left(g_{n}+g_{s}\right) u_{2, k}^{2}+\left(g_{n}-g_{s}\right) u_{1, k}^{2}\right] u_{3, k} & \text { in }(-k, k), \\ -g_{s} u_{1, k} u_{2, k}^{2}=\left(\lambda^{k}-\mu^{k}\right) u_{3, k} & \\ u_{j, k}( \pm k)=0, u_{j, k}(x)>0 \text { for } x \in(-k, k), j=1,2,3, & \end{cases}
$$

where $\lambda^{k}, \mu^{k}$ are two Lagrange multipliers.

Let us collect some properties of $u_{j, k}$ : we have $u_{j, k}(x)>0$ in $(-k, k), u_{j, k}$ is even and decreasing. For $k \geq k_{0}, E_{0}^{k} \leq-C<0$ by Corollary 2.1, and $\left\|u_{j, k}\right\|_{H^{1}\left(I_{k}\right)} \leq C$ for some $\mathrm{C}$ (independent of $k \geq k_{0}$ ). 
Thus we can take a subsequence of $k \rightarrow+\infty$ such that $u_{j, k} \rightarrow u_{j}$ uniformly in $\mathbb{R}$ where $u_{j} \in H^{1}(\mathbb{R}), u_{j} \geq 0$ and $u_{j}$ is decreasing.

Since $u_{j} \in H^{1}(\mathbb{R})$ and $u_{j}$ is decreasing, we see that $u_{j}(x) \rightarrow 0$ as $|x| \rightarrow+\infty$. Thus, for any $\delta>0$, we can find $R_{\delta}>0$ such that for $|x| \geq R_{\delta}, u_{j}(x)<\delta / 2$. As a consequence of decreasing property of $u_{j, k}$, we can find $k_{0}$ such that

$$
u_{j, k}(x) \leq u_{j, k}\left(R_{\delta}\right)<\delta \text {, for }|x|>R_{\delta}, k \geq k_{0} .
$$

Since $u_{j, k} \rightarrow u_{j}$ in $L^{p}\left(-R_{\delta}, R_{\delta}\right)$ and from (4.2) we see that for $p>2, k \geq k_{0}$, $\int_{|x|>R_{\delta}} u_{j, k}^{p} \leq \delta^{p-2}$, we can conclude that $u_{j, k} \rightarrow u_{j}$ in $L^{p}(\mathbb{R})$ for $p>2$.

The main difficulty is to show that $u_{j, k} \rightarrow u_{j}$ strongly in $L^{2}(\mathbb{R})$. If so, then $\left(u_{1}, u_{2}, u_{3}\right)$ satisfies the constraint (1.5)-(1.7) and is a minimizer of the minimization problem (1.8). By the same arguments as those of Claim 1 and Claim 2, we can show that $u_{j}>0$. So it remains to prove the strong convergence in $L^{2}(\mathbb{R})$. We proceed in a few claims.

Claim 3. $\lim _{k \rightarrow+\infty}\left(\lambda^{k}-\mu^{k}\right) \geq 0, \lim _{k \rightarrow+\infty} \lambda^{k} \geq 0, \lim _{k \rightarrow+\infty}\left(\lambda^{k}+\mu^{k}\right) \geq 0$.

In fact, suppose $\lim _{k \rightarrow+\infty}\left(\lambda^{k}+\mu^{k}\right)<-C<0$. Then from the equation for $u_{1, k}$, we see that $u_{1, k}$ satisfies

$$
u_{1, k}^{\prime \prime}+\frac{C}{4} u_{1, k} \leq 0, u_{1, k}(x)>0 \quad \text { in }(-k, k) .
$$

But for $k$ large, by Sturm Comparison theorem, $u_{1, k}$ must change signs in $(-\sqrt{C} \pi, \sqrt{C} \pi)$, which is a contradiction to the fact that $u_{1, k}(x)>0$ in $(-k, k)$.

The other cases can be dealt with similarly.

Claim 4. There exists a positive constant $C>0$ such that

$$
\lambda^{k} N+\mu^{k} M \geq C>0
$$

In fact, by integrating by parts, we obtain that

$$
\lambda^{k} \int_{I_{k}}\left(\sum_{i=1}^{3} u_{i, k}^{2}\right)+\mu^{k} \int_{I_{k}}\left(u_{1, k}^{2}-u_{3, k}^{2}\right) \geq-4 \mathbb{E}^{k}\left(u_{1, k}, u_{2, k}, u_{3, k}\right) \geq C>0
$$

for $k$ large.

Claim 5. There exists $C>0$ such that $\lambda^{k} \geq c_{0}>0$ and $\int_{I_{k}} u_{2, k}^{2} \rightarrow \int_{I_{k}} u_{2}^{2}$ as $k \rightarrow+\infty$.

From Claim 3, we deduce that $\lim _{k \rightarrow+\infty}\left(\lambda^{k}-\left|\mu^{k}\right|\right) \geq 0$. By Claim 4, we have $\lambda^{k} \geq C>0$.

From the equation for $u_{2, k}$ and using (4.2), we have that $u_{2, k}$ satisfies

$$
u_{2, k}^{\prime \prime}-\frac{C}{4} u_{2, k} \geq 0, \quad \text { for }|x|>R, k \geq k_{0}
$$

where $R$ is a fixed large number. Since $u_{2, k}( \pm k)=0$ and by comparison principle, we deduce that

$$
u_{2, k}(x) \leq u_{2, k}(R) e^{-\frac{\sqrt{c_{0}}}{4}|x-R|} \leq C e^{-\frac{\sqrt{C}}{4}|x|} .
$$


Note that $R>R_{\delta}$ only depends on $C$. Thus we conclude that $u_{2, k}$ has exponential decay. So $\int_{I_{k}} u_{2, k}^{2} \rightarrow \int_{\mathbb{R}} u_{2}^{2}$.

Since $\lambda^{k} \geq C>0$, we see that either $\lambda^{k}+\mu^{k} \geq c_{0} / 2$ or $\lambda^{k}-\mu^{k} \geq C / 2$. Let us assume that $\lambda^{k}+\mu^{k} \geq C / 2$. Then by the same proof as Claim 5 , we have

Claim 6. Assuming that $\lambda^{k}+\mu^{k} \geq \frac{C}{2}>0$, then $\int_{I_{k}} u_{1, k}^{2} \rightarrow \int_{\mathbb{R}} u_{1}^{2}$ as $k \rightarrow+\infty$.

Now it remains to show that $\int_{I_{k}} u_{3, k}^{2} \rightarrow \int_{\mathbb{R}} u_{3}^{2}$. Suppose not. By Claim 3, we may assume that $\lim _{k \rightarrow+\infty}\left(\lambda^{k}-\mu^{k}\right)=0$. In fact, if $\lim _{k \rightarrow+\infty}\left(\lambda^{k}-\mu^{k}\right) \geq$ $C>0$, then similar arguments as in Clam 5 show that $u_{3, k}$ has exponential decay and hence $\int_{\mathbb{R}} u_{3, k}^{2} \rightarrow \int_{\mathbb{R}} u_{3}^{2}$ which contradicts to our assumption. (Since $\lambda^{k} \geq C>0, \lambda^{k}+\mu^{k} \geq \frac{C}{2}>0, u_{1, k}$ and $u_{2, k}$ have exponential decay.)

Thus we may also assume that

$$
\lim _{k \rightarrow+\infty}\left(\lambda^{k}-\mu^{k}\right)=0 .
$$

Claim 7. $u_{1} u_{2}^{2} \equiv 0$ and $u_{3} \equiv 0$.

Using (4.8), we see that the limit $u_{3}$ satisfies

$$
u_{3}^{\prime \prime}-\left(g_{n}+g_{s}\right) u_{3}^{3}-\left[\left(g_{n}+g_{s}\right) u_{2}^{2}+\left(g_{n}-g_{s}\right) u_{1}^{2}\right] u_{3}-g_{s} u_{1} u_{2}^{2}=0 \text { in } \mathbb{R} \text {. }
$$

Integrating from 0 to $x$, we obtain that $\left|u_{3}^{\prime}(x)\right| \geq C\left|\int_{0}^{x} u_{1} u_{2}^{2}\right|$. Since $\left\|u_{3}\right\|_{H^{1}} \leq$ $C$, we derive that $u_{3} \equiv 0$ and $u_{1} u_{2}^{2} \equiv 0$.

If both $u_{1} \equiv 0, u_{2} \equiv 0$, we then derive that $N=-M$ (since $u_{1, k} \rightarrow$ $u_{1}, u_{2, k} \rightarrow u_{2}$ strongly in $L^{2}(\mathbb{R})$ ), which is impossible.

There are two cases to be considered.

Case 1. $u_{2}>0, u_{1} \equiv 0$.

By Claim $5, \lambda^{k} \geq C>0$. Since $u_{1}=u_{3} \equiv 0$, from (4.2), we see that $\left|\left(g_{n}+g_{s}\right) u_{1, k}^{2}+\left(g_{n}+g_{s}\right) u_{3, k}^{2}-2 g_{s} u_{1, k} u_{3, k}\right| \rightarrow 0$ uniformly in $\mathbb{R}$. Hence from the equation for $u_{2, k}$ and Claim 7 , we see that $u_{2, k}$ satisfies

$$
u_{2, k}^{\prime \prime}-g_{n} u_{2, k}^{3} \geq \frac{c_{0}}{2} u_{2, k}, u_{2, k}>0 \quad \text { in }(-k, k), u_{2, k}( \pm k)=0 .
$$

Using the equation for $u_{3, k}$ and (4.8), we see that $u_{3, k}$ satisfies

$$
u_{3, k}^{\prime \prime}-g_{n} u_{2, k}^{2} u_{3, k} \leq \frac{C}{4} u_{3, k}, u_{3, k}>0 \quad \text { in }(-k, k), u_{3, k}( \pm k)=0 .
$$

Multiplying (4.10) by $u_{3, k}$ and (4.11) by $u_{2, k}$ and then integrating over $(-k, k)$, we obtain a contradiction.

Case 2. $u_{1}>0, u_{2} \equiv 0$.

In this case, we observe that $u_{1}$ satisfies

$$
u_{1}^{\prime \prime}-\left(g_{n}+g_{s}\right) u_{1}^{3}=2 \lambda^{0} u_{1} \quad \text { in } \mathbb{R}, u_{1} \in H^{1}(\mathbb{R})
$$

where $\lim _{k \rightarrow+\infty} \lambda^{k}=\lim _{k \rightarrow+\infty} \mu^{k}=\lambda^{0}>0$. 
On the other hand, $\frac{u_{2, k}(x)}{u_{2, k}(0)} \rightarrow \hat{u}_{2}(x)$ which satisfies

$$
\hat{u}_{2}^{\prime \prime}-\left(g_{n}+g_{s}\right) u_{1}^{2} \hat{u}_{2}=\lambda^{0} \hat{u}_{2} .
$$

It is easy to see that $0<\hat{u}_{2} \leq 1$ since $\hat{u}_{2}(0)=1$. From (4.12) and (4.13), we obtain then

$$
\lambda^{0} \int_{\mathbb{R}} u_{1} \hat{u}_{2}=0
$$

which is impossible.

In conclusion, we have proved that as $k \rightarrow+\infty, \int_{I_{k}} u_{j, k}^{2} \rightarrow \int_{\mathbb{R}} u_{j}^{2}$ for $j=1,2,3$.

This completes the proof of Theorem 1.1.

\section{Applications to $2 \times 2$ BEC system}

Our proof of Theorem 1.1 can also be applied to obtain ground states for the two-component Bose-Einstein system. Such a system appears in a mixture of BEC of different hyperfine states confined under different kinds of traps [19]. A theoretical study was proposed by the following coupled nonlinear Schrödinger equation [8].

Let

$$
\mathbb{E}_{\beta}(u, v)=\frac{1}{2} \int_{\mathbb{R}}\left(\left|u^{\prime}\right|^{2}+\left|v^{\prime}\right|^{2}\right)-\frac{1}{4} \int_{\mathbb{R}}\left(\mu_{1}|u|^{4}+\mu_{2}|v|^{4}+2 \beta u^{2} v^{2}\right),
$$

where $\mu_{1}>0$ and $\mu_{2}$ are positive constants, and $\beta \in \mathbb{R}$ is the coupling constant.

Let $N>0$. Consider the constrained minimization problem

$$
\inf \left\{E_{\beta}(u, v) \mid \int_{\mathbb{R}}\left(|u|^{2}+|v|^{2}\right)=N\right\} .
$$

A nontrivial solution to (5.2) is called a ground state which satisfies the following Euler-Lagrange equation

$$
\left\{\begin{array}{l}
u^{\prime \prime}+\mu_{1} u^{3}+\beta u v^{2}=\lambda u \\
v^{\prime \prime}+\mu_{2} v^{3}+\beta u^{2} v=\lambda v \\
u, v \in H^{1}(\mathbb{R})
\end{array}\right.
$$

Then we have

Theorem 5.1. If $\beta>\max \left\{\mu_{1}, \mu_{2}\right\}$, then (5.2) can be achieved by $\left(u_{0}, v_{0}\right)$ such that $u_{0}>0, v_{0}>0$. Furthermore, we have

$$
u_{0}=\sqrt{\frac{\beta-\mu_{2}}{\beta^{2}-\mu_{1} \mu_{2}}} w, \quad v_{0}=\sqrt{\frac{\beta-\mu_{1}}{\beta^{2}-\mu_{1} \mu_{2}}} w,
$$

where $w$ is the unique homoclinic solution of

$$
w^{\prime \prime}-\lambda w+w^{3}=0 .
$$


On the other hand, if we consider the following minimization problem

$$
\inf \left\{\left.E_{\beta}(u, v)\left|\int_{\mathbb{R}}\right| u\right|^{2}=N_{1}, \int_{\mathbb{R}}|v|^{2}=N_{2}\right\},
$$

where $N_{1}>0, N_{2}>0$, then we have

Theorem 5.2. If $\beta>0$ then (5.6) can be achieved by $\left(u_{0}, v_{0}\right)$ such that $u_{0}>$ $0, v_{0}>0$ and satisfy

$$
\left\{\begin{array}{l}
u^{\prime \prime}+\mu_{1} u^{3}+\beta u v^{2}=\lambda_{1} u \quad \text { in } \mathbb{R}, \\
v^{\prime \prime}+\mu_{2} v^{3}+\beta u^{2} v=\lambda_{2} v \quad \text { in } \mathbb{R}, \\
u, v \in H^{1}(\mathbb{R})
\end{array}\right.
$$

for some $\lambda_{1}>0, \lambda_{2}>0$.

We remark that the minimization problems (5.2), (5.6) arise naturally in the study of standing waves of the coupled Gross-Pitaevskii equations, i.e., the coupled nonlinear Schrödinger equations,

$$
\left\{\begin{array}{l}
-i \frac{\partial}{\partial t} \Phi_{1}=\Delta \Phi_{1}+\mu_{1}\left|\Phi_{1}\right|^{2} \Phi_{1}+\beta\left|\Phi_{2}\right|^{2} \Phi_{1}, \\
-i \frac{\partial}{\partial t} \Phi_{2}=\Delta \Phi_{2}+\mu_{2}\left|\Phi_{2}\right|^{2} \Phi_{2}+\beta\left|\Phi_{1}\right|^{2} \Phi_{2}, \\
\Phi_{j}=\Phi_{j}(y, t) \in \mathbb{C}, \quad j=1,2,
\end{array}\right.
$$

where $\mu_{1}, \mu_{2}$ are positive constants, and $\beta$ is a coupling constant.

For any solutions to (5.8), the following two integrals are conserved

$$
\int_{\mathbb{R}^{d}}\left|\Phi_{1}(x, t)\right|^{2}=\int_{\mathbb{R}^{d}}|\Phi(x, 0)|^{2}, \int_{\mathbb{R}^{d}}\left|\Phi_{2}(x, t)\right|^{2}=\int_{\mathbb{R}^{d}}\left|\Phi_{2}(x, 0)\right|^{2} .
$$

Thus it is natural to consider problems (5.2)-(5.6).

System (5.8) arises in the Hartree-Fock theory for a double condensate, i.e. a binary mixture of Bose-Einstein condensates in two different hyperfine states $|1\rangle$ and $|2\rangle([8])$.

To obtain solitary wave solutions of the system (5.8), we set $\Phi_{1}(x, t)=$ $e^{i \lambda_{1} t} u(x), \Phi_{2}(x, t)=e^{i \lambda_{2} t} v(x)$, and the system (5.8) is transformed to an elliptic system given by

$$
\left\{\begin{array}{l}
\Delta u-\lambda_{1} u+\mu_{1} u^{3}+\beta u v^{2}=0 \quad \text { in } \mathbb{R}^{d}, \\
\Delta v-\lambda_{2} v+\mu_{2} v^{3}+\beta u^{2} v=0 \quad \text { in } \mathbb{R}^{d}, \\
u, v \in H^{1}\left(\mathbb{R}^{d}\right)
\end{array}\right.
$$

where $\lambda_{1}, \lambda_{2}, \mu_{1}, \mu_{2}>0$ are positive constants and $\beta \neq 0$ is a coupling constant.

The existence of least energy solution to $(5.10)$ is studied in $[2,15,24]$. In $[2,4,16,17,24]$, the existence of bound states [i.e., solutions to (5.10)] when $\beta>0$ is proved. Note that the minimization problems (5.2) and (5.6) are different from the minimization problems in [2] and [15].

Proof of Theorem 5.1. Following the proof of Theorem 1.1, and using Schwarz symmetrization, we can show the existence of minimizer $\left(u_{0}, v_{0}\right)$ such that $u_{0} \in H^{1}(\mathbb{R}), v_{0} \in H^{1}(\mathbb{R}), u_{0} \geq 0, v_{0} \geq 0$ and satisfies (5.3). (Here as in the proof of Theorem 1.1, we have to first work on a bounded domain $(-k, k)$ for $k$ large. Then we show the $L^{2}$-strong convergence of the sequence.) 
The difficulty is to show that $u_{0} \not \equiv 0, v_{0} \not \equiv 0$.

We argue by way of contradiction. Suppose that $v_{0} \equiv 0$. Then $u_{0} \not \equiv 0$ and $u_{0}$ is a positive solution of

$$
u^{\prime \prime}+\mu_{1} u^{3}+\beta v^{2} u=\lambda u \text { in } \mathbb{R} .
$$

Set $u=u_{0}+\varepsilon_{1} \varphi_{1}, v=\varepsilon_{2} \varphi_{2}$, with $\varepsilon_{1}>0, \varepsilon_{2}>0, \varphi_{1} \in H^{1}(\mathbb{R}), \varphi_{2} \in$ $H^{1}(\mathbb{R})$ such that

$$
\int_{\mathbb{R}}\left(|u|^{2}+|v|^{2}\right)=\int_{\mathbb{R}}|u|^{2}=N
$$

namely

$$
2 \varepsilon_{1} \int_{\mathbb{R}} u_{0} \varphi_{1}+\varepsilon_{1}^{2} \int_{\mathbb{R}}\left|\varphi_{1}\right|^{2}+\varepsilon_{2}^{2} \int_{\mathbb{R}}\left|\varphi_{2}\right|^{2}=0 .
$$

We can choose $\varphi_{1}$ such that $\int_{\mathbb{R}} u_{0} \varphi_{1}<0$, then for fixed $\varepsilon_{2}>0$ small, $\varphi_{2} \not \equiv 0$ there exists $\varepsilon_{1}>0$ so that (5.11) holds and $\varepsilon_{1}=O\left(\varepsilon_{2}^{2}\right)$.

Using (5.11) and $\varepsilon_{1}=O\left(\varepsilon_{2}^{2}\right)$ we have

$$
\begin{aligned}
\mathbb{E}_{\beta}(u, v)= & \frac{1}{2} \int_{\mathbb{R}}\left(\left|u_{0}^{\prime}+\varepsilon_{1} \varphi_{1}^{\prime}\right|^{2}+\varepsilon_{2}^{2}\left|\varphi_{2}^{\prime}\right|^{2}\right) \\
& -\frac{1}{4} \int_{\mathbb{R}}\left(\mu_{1}\left|u_{0}+\varepsilon_{1} \varphi_{1}\right|^{4}+\mu_{2} \varepsilon_{2}^{4}\left|\varphi_{2}\right|^{4}+2 \beta \varepsilon_{2}^{2}\left(u_{0}+\varepsilon_{1} \varphi_{1}\right)^{2} \varphi_{2}^{2}\right) \\
= & \mathbb{E}_{\beta}\left(u_{0}, 0\right)+\varepsilon_{1} \int_{\mathbb{R}}\left(u_{0}^{\prime} \varphi_{1}^{\prime}-\mu_{1} u_{0}^{3} \varphi_{1}\right)+O\left(\varepsilon_{1}^{2}\right) \\
& +\varepsilon_{2}^{2}\left(\frac{1}{2} \int_{\mathbb{R}}\left|\varphi_{2}^{\prime}\right|^{2}-\frac{\beta}{2} \int_{\mathbb{R}} u_{0}^{2} \varphi_{2}^{2}\right)+O\left(\varepsilon_{2}^{3}\right) \\
= & \mathbb{E}_{\beta}\left(u_{0}, 0\right)-\varepsilon_{1} \lambda \int_{\mathbb{R}} u_{0} \varphi_{1}+O\left(\varepsilon_{1}^{2}\right)+\varepsilon_{2}^{2}\left(\frac{1}{2} \int_{\mathbb{R}}\left|\varphi_{2}^{\prime}\right|^{2}-\frac{\beta}{2} \int_{\mathbb{R}} u_{0}^{2} \varphi_{2}^{2}\right)+O\left(\varepsilon_{2}^{3}\right) \\
= & \mathbb{E}_{\beta}\left(u_{0}, 0\right)+\varepsilon_{2}^{2}\left(\frac{1}{2} \int_{\mathbb{R}}\left(\left|\varphi_{2}^{\prime}\right|^{2}+\lambda \varphi_{2}^{2}\right)-\frac{\beta}{2} \int_{\mathbb{R}} u_{0}^{2} \varphi_{2}^{2}\right)+O\left(\varepsilon_{2}^{3}\right) .
\end{aligned}
$$

Note that the first eigenvalue of

$$
-\phi^{\prime \prime}+\lambda \phi=\nu u_{0}^{2} \phi
$$

is $\mu_{1}$. Thus if $\beta>\mu_{1}$, then we can choose $\varphi_{2}$ such that

$$
\int_{\mathbb{R}}\left(\left|\varphi_{2}^{\prime}\right|^{2}+\lambda \varphi_{2}^{2}\right)-\beta \int_{\mathbb{R}} u_{0}^{2} \varphi_{2}^{2}<0
$$

and therefore $\mathbb{E}_{\beta}(u, v)<\mathbb{E}_{\beta}\left(u_{0}, 0\right)$, which deduces that $v_{0} \not \equiv 0$.

Similarly, if $\beta>\mu_{2}$, then $u_{0}>0$. Therefore we have proved the first part of Theorem 5.1.

To prove the last part, we consider the set $I=\left\{u_{0}>c v_{0}\right\}$, where $c=$ $\sqrt{\frac{\beta-\mu_{2}}{\beta-\mu_{1}}}$. From the equation (5.3), we have

$$
\left.\left(u_{0}^{\prime} v_{0}-u_{0} v_{0}^{\prime}\right)\right|_{\partial I}+\int_{I}\left[\left(\mu_{1} u_{0}^{3}+\beta v_{0}^{2} u_{0}\right) v_{0}-\left(\mu_{2} v_{0}^{3}+\beta u_{0}^{2} v_{0}\right) u_{0}\right]=0 .
$$


Note that on $I$,

$\int_{I}\left[\left(\mu_{1} u_{0}^{3}+\beta v_{0}^{2} u_{0}\right) v_{0}-\left(\mu_{2} v_{0}^{3}+\beta u_{0}^{2} v_{0}\right) u_{0}\right]=\left(\mu_{2}-\beta\right) \int_{I} u_{0} v_{0}\left(u_{0}^{2}-c^{2} v_{0}^{2}\right) \leq 0$.

On the other hand,

$$
\left.\left(u_{0}^{\prime} v_{0}-u_{0} v_{0}^{\prime}\right)\right|_{\partial I} \leq 0 .
$$

Thus $I$ must be an empty set. So $u_{0} \leq c v_{0}$. Similarly we can prove that $u_{0} \geq c v$.

So $u_{0}=c v_{0}$. This proves (5.4).

Proof of Theorem 5.2. This follows easily from the proof of Theorem 1.1. Note that because of the constraints, we obtain $u_{0}>0, v_{0}>0$.

\section{Acknowledgments}

Part of this work was done while D. Cao was visiting Institute of Mathematical Sciences, the Chinese University of Hong Kong. D. Cao was partially supported by the Key Project of NSF (No. 10631030) and Science Fund for Creative Research Groups of NSFC (No. 10721101). The research of JW is partially supported by GRF of HKRGC. We thank the referee for carefully reading the manuscript and many critical suggestions.

\section{References}

[1] Anderson, M.H., Ensher, J.R., Mathews, M.R., Wieman, C.E., Cornell, E.A.: Observation of Bose-Einstein condensation in a dilute atomic vapor. Science 269, 198 (1995)

[2] Ambrosetti, A., Colorado, E.: Bound and ground states of coupled nonlinear Schrodinger equations. C. R. Math. Acad. Sci. Paris 342, 453-458 (2006)

[3] Barrett, M.D., Sauer, J.A., Chapman, M.S.: All-optical formation of an atomic Bose-Einstein condensate. Phys. Rev. Lett. 87, 010404 (2001)

[4] Bartsch, T., Wang, Z.-Q., Wei, J.: Bound states for a coupled Schrödinger system. J. Fixed. Point. Theory. Appl. 2, 353-367 (2007)

[5] Bradley, C.C., Sackett, C.A., Tollett, J.J., Hulet, R.G.: Evidence of BoseEinstein condensation in an atomic gas with attractive interaction. Phys. Rev. Lett. 75, 1687 (1995)

[6] Dalfovo, F., Giorgini, S., Pitaevskii, L.P., Stringari, S.: Theory of Bose-Einstein condensation in trapped gases. Rev. Mod. Phys. 71, 463-512 (1999)

[7] Davis, K.B., Mewes, M.-O., Andrews, M.R., van Druten, N.J., Durfee, D.D., Kurn, D.M., Ketterle, W.: Bose-Einstein condensation in a gas of sodium atoms. Phys. Rev. Lett. 75, 3969 (1995)

[8] Esry, B.D., Greene, C.H., Burke, J.P. Jr., Bohn, J.L.: Hartree-Fock theory for double condensates. Phys. Rev. Lett. 78, 3594-3597 (1997) 
[9] Gerdjikov, V.S., Kostov, N.A., Valchev, T.I.: Solutions of multi-component NLS models and spinor Bose-Einstein condensates. Physica D. doi:10.1016/j.physd. 2008.06.007

[10] Görlitz, A., Gustavson, T.L., Leanhardt, A.E., Löw, E., Chikkatur, A.P., Gupta, S., Inouye, S., Pritchard, D.E., Katterle, W.: Sodium Bose-Einstein condensates in the $F=2$ state in a large-volume optical trap. Phys. Rev. Lett. 90, 090401 (2003)

[11] Gross, E.P.: Nuovo, structure of a quantized vortex in boson systems. Nuovo Cimento 20, 454-477 (1961)

[12] Ho, T.L.: Spinor Bose condensates in optical traps. Phys. Rev. Lett. 81, 742745 (1998)

[13] Law, C.K., Pu, H., Bigelow, N.P.: Quantum spins mixing in spinor Bose-Einstein condensates. Phys. Rev. Lett. 81, 5257-5261 (1998)

[14] Lieb, E., Loss, M.: Analysis. Amer. Math. Soc., Providence (1996)

[15] Lin, T.-C., Wei, J.-C.: Ground state of $N$ coupled nonlinear Schrödinger equations in $R^{n}, n \leq 3$. Commun. Math. Phys. 255(3), 629-653 (2005)

[16] Lin, T.-C., Wei, J.-C.: Spikes in two coupled nonlinear Schrödinger equations. Ann. Inst. Poincare Anal. Non-Lin. 22, 403-439 (2005)

[17] Maia, L.A., Montefusco, E., Pellacci, B.: Positive solutions for a weakly coupled nonlinear Schrödinger system. J. Differ. Equ. 229(2), 743-767 (2006)

[18] Miesner, H.J., Stamper-Kurn, D.M., Stenger, J., Inouye, S., Chikkatur, A.P., Ketterle, W.: Observation of metastable states in spinor Bose-Einstein condensates. Phys. Rev. Lett. 82, 2228-2231 (1999)

[19] Myatt, C.J., Burt, E.A., Ghrist, R.W., Cornell, E.A., Wieman, C.E.: Production of two overlapping Bose-Einstein condensates by sympathetic cooling. Phys. Rev. Lett. 78, 586-589 (1997)

[20] Ohmi, T., Machida, K.: Bose-Einstein condensation with internal degrees of freedom in alkali atom gases. J. Phys. Soc. Jpn. 67, 1822-1825 (1998)

[21] Pitaevskii, L.P.: Vortex lines in an imperfect Bose gas. Soviet Phys. JETP 13, 451-454 (1961)

[22] Stamper-Kurn, D.M., Andrews, M.R., Chikkatur, A.P., Inouye, S., Miesner, H.-J., Stenger, J., Ketterle, W.: Optical confinement of a Bose-Einstein condensate. Phys. Rev. Lett. 80, 2027-2030 (1998)

[23] Stenger, J., Inouye, S., Stamper-Kurn, D.M., Miesner, H.-J., Chikkatur, A.P., Ketterle, W.: Spin domains in ground state Bose-Einstein condensates. Nature 396, 345-348 (1998)

[24] Sirakov, B.: Least energy solitary waves for a system of nonlinear Schrodinger equations. Commun. Math. Phys. 271, 199-221 (2007) 
[25] Weinstein, M.I.: Nonlinear Schrödinger equations and sharp interpolation estimates. Commun. Math. Phys. 87, 567-576 (1983)

Daomin Cao

Institute of Applied Mathematics,

AMSS, Chinese Academy of Sciences,

Beijing 100190,

People's Republic of China

e-mail: dmcao@amt.ac.cn

I-Liang Chern

Department of Mathematics,

National Taiwan University,

Taipei 106,

Taiwan, ROC

e-mail: chern@math.ntu.edu.tw

Jun-Cheng Wei

Department of Mathematics,

Chinese University of Hong Kong,

Shatin, Hong Kong,

People's Republic of China

e-mail: wei@math.cuhk.edu.hk

Received: 25 June 2010.

Accepted: 14 January 2011. 